5th International Conference on Fatigue and Transportation

Local: Esplanade Hotel, Fremantle, Western Australia, Austrália

Data: 11 a 15 de março de 2002

Tema: Coping with the 24 hour society. Fatigue management alternatives to prescriptive hours of service. "Counter measures: detection technology, naps and drugs" Informações: Laurence Hartley, Conference Convenor, Institute for Research in Safety \& Transport Psychology, Murdoch University, Western Australia 6150

Fax: 61893606492 hartley@socs.murdoch.edu.au http://www.congresswest.com.au/FATIGUE2002 http://www.socs.murdoch.edu.au/irst/irst.htm

4th International Congress on Vegetarian Nutrition Local: Loma Linda, California, Estados Unidos Data: 17 a 22 de março de 2002 Informações: Department of Nutrition, School of Public Health, Loma Linda University,

Loma Linda, CA 92350

ICVN@sph.llu.edu

I Seminário Internacional de Engenharia de Saúde Pública

Local: Recife, Pernambuco, Brasil

Data: 18 a 20 de março de 2002

Informações: FUNASA, Departamento de Engenharia de Saúde Pública. SAS Quadra 4, Bloco N, 10o andar, sala 1003, Brasília, DF 70070-040, Brasil

Tel: (61) 3146278; Fax: (61) 2260326

densp.seminario@funasa.gov.br

Epi 2002. V Congresso Brasileiro

\section{de Epidemiologia}

Local: Pontifícia Universidade Católica do Paraná,

Curitiba, Paraná, Brasil

Data: 23 a 27 de março de 2002

Tema: A epidemiologia na promoção da saúde Informações: Secretaria Executiva, PJ Eventos Feiras e Congressos. Rua José Risseto, 1023, Santa Felicidade, Curitiba, PR 82015-010, Brasil Tel: +55 41 3721177; Fax: +55 413741094 pjeventos@pjeventos.com.br http://www.pjeventos.com.br/epi2002

3rd International Conference on Emerging Infectious Diseases (ICEID2002)

Local: Hyatt Regency Hotel, Atlanta,

Georgia, Estados Unidos

Data: 24 a 27 de março de 2002

Informações: Charles Schable

cas1@cdc.gov

http://www.cdc.gov/ICEID/index.htm
III Conferência Latino-Americana de promoção e Educação em Saúde e I Encontro LatinoAmericano de Educação Popular em Saúde Local: São Paulo, São Paulo, Brasil

Data: março de 2002

Informações: UIPES/ORLA/Sub-região Brasil. Rua Voluntários da Pátria, 169 - 13o andar, 22270-000, Botafogo, Rio de Janeiro, RJ, Brasil Tel: (21) 22751202/(21) 25368856 Fax: (21) 25424149/(21) 22862638

vllima.orla@openlink.com.br

\section{3rd World Congress on Neurological} Rehabilitation

Local: Veneza, Italy

Data: 3 a 6 de abril de 2002

Informações: Organizing Secretariat

Fax: +3906 84498332

neurorehab2002@aristea.com

\author{
The 8th Annual Qualitative Health \\ Resesrch Conference \\ Local: The Banff Centre for Conference, \\ Banff, Alberta, Canadá \\ Data: 4 a 6 de abril de 2002 \\ Informações: ualberta.ca/ iiqm
}

\section{Congresso Português de Cardiologia}

Local: Vilamoura, Portugal

Data: 13 a 17 de abril de 2002

Informações: Sociedade Portuguesa

de Cardiologia. Campo Grande, 28, 13o andar.

1700-093, Lisboa, Portugal

Tel: (21) 7817634; Fax: (21) 7931095

congresso@mail.spc.pt

\section{Experimental Biology 2002}

Local: New Orleans, Lousiana, Estados Unidos Data: 20 a 24 de abril de 2002

Informações: Jean Lash, Exhibit Manager Federation of American Societies for Experimental Biology, 9650 Rockville Pike, Bethesda, MD 20814-3998

Tel: 301 530-7009; Fax: 301 530-7014

jlash@faseb.org

http://www.faseb.org/meetings

\section{8o Enftec: Enfermagem no século XXI} Reflexôes e Visões

Local: Centro de Conferência e Exposições Amcham, São Paulo, São Paulo, Brasil

Data: 22 a 25 de abril de 2002

Informações: GMC Bureau de Eventos

Tel/Fax: (11) 3257-8248 DAS 9h ÀS 16h

gmceventos@attglobal.net

http://www.cee8ago.org.br 


\author{
6th World Conference on Injury \\ Prevention and Control \\ Local: Montreal, Quebec, Canadá \\ Data: 12 a 15 de maio de 2002 \\ Informações: 511, place d'Armes, \#600, \\ Montreal QC H2Y sW7, Canada \\ Tel: 514848 1133; Fax: 5142886469 \\ trauma@coplanor.qc.ca \\ http://www.trauma2002.com
}

\section{7th World Congress of the International Federation of Environmental Health \\ Local: San Diego, California, Estados Unidos \\ Data: 20 a 24 de maio de 2002 \\ Informações: Diane Evans \\ Tel: (831) 4542022 \\ diane.evans@co.santacruz.ca.us \\ http://www.ifeh2002.org}

6th European Congress of Clinical Gerontology

Local: Moscow, Russia

Data: junho de 2002

Informações: Professor L. B. Lazebnik

lazebnik@aha.ru

\section{Cruzando Fronteras en América Latina.}

Tercer Congreso Europeo de Latinoamericanistas

Local: Amsterdam, Holanda

Data: 3 a 6 de julho de 2002

Informações: CEISAL 2002, c/o CEDLA,

Keizersgracht 395-397, 1016 EK Amsterdam, Holanda

Fax: (+31 20) 6255127

ceisal02@cedla.uva.nl

http:// www.cedla.uva.nl/ceisal-2002

\section{Internacional AIDS Conference}

Local: Barcelona, Espanha

Data: 7 a 12 de julho de 2002

Tema: Knowledge and Commitment for Action

Informações: Barcelona Secretariat.

Pomaret 21, 08017, Barcelona, Spain

Tel: +34 93 2540555; Fax: +34 932540575

aids2002@aids2002.com

http://www.aids2002.com

The IEA XVI World Congress of Epidemiology

Local: Montreal, Canadá

Data: 18 a 22 de agosto de 2002

Informações: IEA2002 Secretariat, Events

International Meeting Planners. 759 Square Victoria,

Suite 300, Montreal, Quebec H2Y257, Canada

Tel: +1.514.286.0855; Fax: +1.514.286.6066

info@eventsintl.com

http://www.iea2002.com

The 11th International Conference

for Science Editors

Local: Beijing, China

Data: 24 a 29 de agosto de 2002

Informações: Secretariat of IFSE-11, c/o Ms. Cuiling

Lan. Center for International Scientific Exchanges,

Chinese Academy of Sciences. No. 52 Sanlihe Road,

Xicheng, Beijing 100864, P.R.China

Tel: 86-10-68597750; Fax: 86-10-68597748

cllan@cashq.ac.cn

http://www.cessp.org.cn/ifse.htm
IV Congresso da Sociedade Brasileira de Doenças Sexualmente Transmissíveis

Local: Manaus, Amazonas, Brasil

Data: 1 a 4 de setembro de 2002

Informações: ORCAL Pesquisas \& Eventos.

Av. Joaquim Nabuco, 352, Centro,

Manaus, AM 69020-031, Brasil

Tel: (92) 622-3377

Tel/Fax: (92) 232-4468/(92) 234-5063

orcal@osite.com.br

http://www.fuam.am.gov.br/congressodst

$5^{\text {th International Conference of the Hospital }}$ Infection Society

Local: Edinburgh International Conference Centre, Edinburgh, Escócia

Data: 15 a 18 de setembro de 2002

Informações: Concorde Services, HIS2002, Unit 4b, 50 Speirs Wharf, Port Dundas, Glasgow G4 9TB,

Scotland, UK.

Tel: +44 (0) 141331 0123; Fax: +44 (0) 1413310234

his@concorde-uk.com

http://www.his2002.co.uk

Twelfth Latin American Congress

of Nutritionists and Dieticians

Local: Caracas, Venezuela

Data: 30 de setembro a 4 de outubro de 2002

Informações: Lesbia Gonzalez, RD, Presidente,

Confederación Latinoamericana de Nutricionistas

y Dietistas. Centro Comercial Uslar Torre de Oficina.

Piso 9 of 94, Montalban, Caracas, Venezuela

Tel: +58 2124427 911; Fax: +58 2124428101

colnutri@cantv.net

http://www.confelanyd.org

Health Research in Rural and Remote Canada: Taking the Next Steps

Local: Saskatoon, Saskatchewan, Canadá

Data: 18 a 21 de outubro de 2002

Informações: Rural Health Research Consortium. 103 Hospital Drive, P.O. Box 120, R.U.H., S7N 0W8

Saskatoon, SK Canada

Tel: (306) 966-7888; Fax: (306) 966-8378

bizetto@sask.usask.ca

http://www.usask.ca/medicine/agmedicine

Biotecnología Habana 2002:

La Agro-Biotecnología en el Nuevo Milenio

Local: Centro de Ingeniería Genética y Biotecnología,

Ciudad de la Habana, Cuba

Data: 24 a 29 de novembro de 2002

Informações: Apartado Postal 6162, La Habana

10600, Cuba

Tel: 53-7-218008; Fax: 53-7-218070

bioagro@cigb.edu.cu

http://bioagro.cibg.edu.cu 